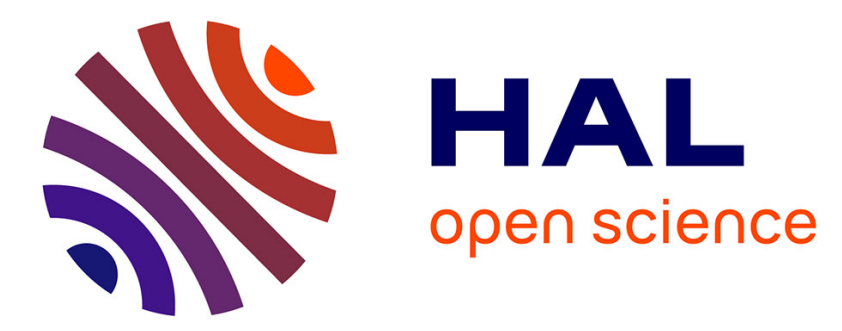

\title{
Achromatic induction: A variational interpretation of Rudd-Zemach's edge integration model
}

Edoardo Provenzi

\section{To cite this version:}

Edoardo Provenzi. Achromatic induction: A variational interpretation of Rudd-Zemach's edge integration model. International Conference on Signal-Image Technology \& Internet-Based Systems, Dec 2013, Kyoto, Japan. 10.1109/SITIS.2013.75 . hal-01093431

\section{HAL Id: hal-01093431 \\ https://hal.science/hal-01093431}

Submitted on 10 Dec 2014

HAL is a multi-disciplinary open access archive for the deposit and dissemination of scientific research documents, whether they are published or not. The documents may come from teaching and research institutions in France or abroad, or from public or private research centers.
L'archive ouverte pluridisciplinaire HAL, est destinée au dépôt et à la diffusion de documents scientifiques de niveau recherche, publiés ou non, émanant des établissements d'enseignement et de recherche français ou étrangers, des laboratoires publics ou privés. 


\title{
Achromatic induction: A variational interpretation of Rudd-Zemach's edge integration model
}

\author{
Edoardo Provenzi \\ Department of Signal and Image Processing \\ Télécom ParisTech, LTCI, CNRS \\ Paris, France \\ edoardo.provenzi@telecom-paristech.fr
}

\begin{abstract}
Induction is the term used to describe the fact that visual perception depends not only on the absolute luminance of a patch in a visual scene, but also on the luminance of the surrounding patches. Induction can be achromatic or chromatic, depending on the presence of only shades of gray, or color, respectively. Achromatic induction can be measured through quite simple psychophysical matching experiments, whose data are well fitted by the so-called edge integration models. In this paper we will examine RuddZemach's edge integration model and we will show that it can be interpreted in terms of a variational framework that shares many features with a phenomenologically-based visual perception theory recently proposed. The relevance of this result is twofold: firstly, it allows interpreting induction in terms of local contrast enhancement, secondly, it provides a validation of the variational framework in terms of quantitative measures, opening the possibility to determine important parameters of this framework.
\end{abstract}

Keywords-Achromatic induction, edge integration models, variational principles, phenomenology of visual perception.

\section{INTRODUCTION}

Human perception of the color of a patch in a visual scene is not determined only by luminance of the patch itself, but also by that of the surrounding patches. This phenomenon is called chromatic induction, to stress the fact that color perception of a patch is induced (and altered) by its surround. What just stated is also true for the so-called achromatic colors, i.e. perceived shades of gray, in which case one talks about achromatic induction.

Following a common nomenclature, we will call lightness the perceived luminance reflected by a non selfluminous patch, while brightness will refer to the perceived luminance emitted by a source of light. Thus, the terms lightness and brightness already incorporate the effect of induction in their definition.

The most elementary example of induction is the simultaneous contrast phenomenon, depicted in Fig. 1: the inner squares have exactly the same luminance, however we perceive them very differently because we are strongly influences by their distinct surrounds.

Induction can be measured through psychophysical experiments. The first quantitative measure of achromatic induction has been performed by $\mathrm{H}$. Wallach in [1]. In his classical experiment, see Fig. 2 (courtesy of [2]), Wallach considered two disks, $T$ and $M$ for Target and Match, embedded in black background $B$, with luminance $D_{T}$ and $D_{M}$, surrounded by two rings of luminance $R_{T}$ and

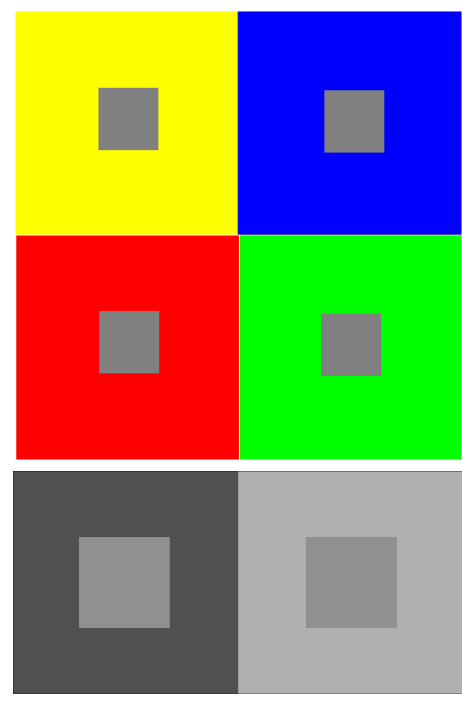

Figure 1. Top: Chromatic simultaneous contrast. Bottom: Achromatic simultaneous contrast.

$R_{M}$, respectively. He showed this configuration to a set of observers adapted to the light condition of a dimly illuminated room keeping $D_{T}$ and $R_{M}$ fixed, using $R_{T}$ as an independent variable that he could fix in every experiment, and $D_{M}$ as a dependent variable that the users could adjust in order to achieve a perceptual match between the two disks $T$ and $M$. Notice that the luminance values selected fall in the scotopic (nearly mesopic) range, to assure that only achromatic stimuli are generated.

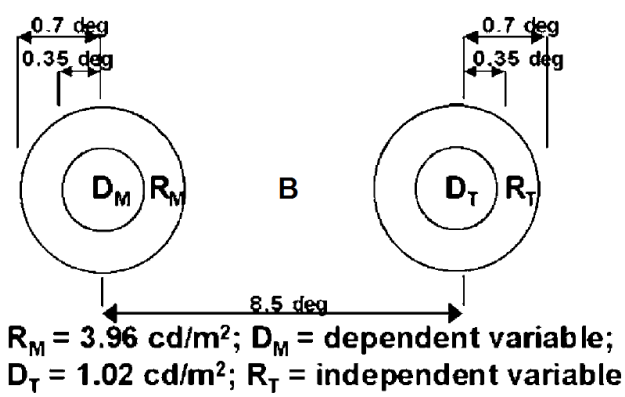

Figure 2. Wallach's classical experiment.

If the luminance of the surrounding rings failed to influence the perception of the achromatic color of the disks, then the match among the two disks would be simply the photometric match, i.e. $D_{M}=D_{T}$, instead 
Wallach found that a fairly good achromatic color match among the two disks was obtained when the ratios between the disk and the ring luminances were identical on the two sides of the display, i.e.

$$
\frac{D_{M}}{R_{M}}=\frac{D_{T}}{R_{T}}
$$

a formula called Wallach's Ratio Rule. By taking the Briggs (decimal) logarithms at both sides and solving for $D_{M}$ we find:

$$
\log D_{M}=\log D_{T}+\log R_{M}-\log R_{T},
$$

thus, according to Wallach's Ratio Rule, the plot of the perceptual match in the plane of coordinates $(x, y)=$ $\left(\log R_{T}, \log D_{M}\right)$ should be a straight line with slope -1 , against the slope 0 that a photometric match would measure.

More recent measures on the classical Wallach's experiment show that this slope is actually between -1 and 0 , as it can be seen in Fig. 3 (adapted from [2]).

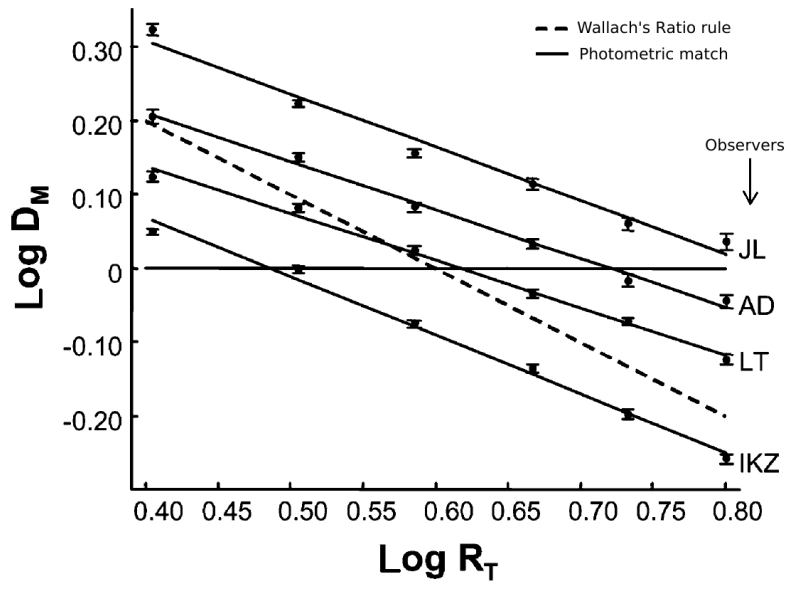

Figure 3. Quantitative measures of Wallach's achromatic color induction experiment for four observers performed in [2]. The best-fit regression line slopes and associated 95\% confidence limits reported in [2] are the following: $-0.639 \pm 0.033$ (LT), $-0.791 \pm 0.034$ (IKZ), $-0.723 \pm 0.047$ (JL), and $-0.657 \pm 0.042(\mathrm{AD})$.

To account for these new psychophysical data, M.E. Rudd and I.K. Zemach have proposed a more sophisticated model than Wallach's. Rudd-Zemach's model will be detailed in Section II. In Section III a generalization of RuddZemach's model to arbitrary spatial configurations and its variational interpretation will be discussed, followed Section IV, in which the relationship with the perceptuallyinspired variational framework of [3] will be analyzed. The interest of this result is twofold: from one side, the variational framework allows understanding induction in terms of local contrast enhancement, on the other side, the psychophysical measures on which Rudd-Zemach's model rely give the first quantitative validation of the variational framework introduced in [3] and can be used to determine important parameters within this framework.

\section{RUDD-ZEMACH'S EDGE INTEGRATION MODEL FOR ACHROMATIC INDUCTION}

Rudd and Zemach in [2] repeated Wallach's experiment adding a non-black background $B$. The experimental setting is described in detail in [2]. As in Wallach's experiment, $D_{T}$ and $R_{M}$ are fixed and the observer's task is to adjust $D_{M}$ to achieve an achromatic color match to the test disk as a function of $R_{T} . R_{T}$ is varied from trial to trial by random sampling from a set of six luminance values spaced equally in units from 2.54 to $6.31 \mathrm{~cd} / \mathrm{m}^{2}$ (0.405 to $0.800 \log c d / m^{2}$ ).

Rudd and Zemach claim that the inspiration for their model of achromatic induction is the Retinex theory of color perception of Land and McCann [4], however, they use a version of Retinex without the so-called threshold and reset mechanisms, see [5] for more details.

Let $L_{i}$ and $L_{j}$ be the luminance of two points $i$ and $j$ in an image, the ratio $\frac{L_{j}}{L_{i}}$ can be decomposed as a chain multiplication of the local luminance ratios at borders encountered along a path connecting $j$ and $i$ :

$$
\frac{L_{j}}{L_{i}}=\prod_{k=i}^{j-1} \frac{L_{k+1}}{L_{k}}
$$

by taking the logarithm at both sides we get

$$
\log \frac{L_{j}}{L_{i}}=\sum_{k=i}^{j-1} \log \frac{L_{k+1}}{L_{k}} .
$$

Rudd and Zemach introduced induction strength weights $w_{k}$, in order to take into account locality of vision, i.e. the different influence of nearby vs. far away luminance, and defined the logarithmic brightness of $i$ as follows:

$$
\Phi_{i} \equiv \sum_{k=i}^{j-1} w_{k-i+1} \log \frac{L_{k+1}}{L_{k}} .
$$

Notice that, if the luminances $L_{k}$ and $L_{k+1}$ are equal, then their ratio does not give any contribution to $\Phi_{i}$. A meaningful contribution to $\Phi_{i}$ is given only by the luminances of points lying at the border of an edge. So, $\Phi_{i}$ represents the summed influence of all the edges present within the spatial surround on the target point, suitably weighted. The index of the weights is such that small values of the index label patches close to $i$ and large values of the index label patches far away from $i$. With this convention, and knowing that induction strength declines with distance [6], we have that $w_{1}>w_{2}>\ldots$, i.e. $\frac{w_{2}}{w_{1}}<1$, and so on.

Rudd and Zemach call their model of achromatic induction 'Weighted Log Luminance Ratio', or WLLR for short. If WLLR were the true model of achromatic induction, then an observer would match the brightness (perceived luminance) of the disks $D_{M}$ and $D_{T}$ of Fig. 2 when this equation holds

$$
w_{1} \log \frac{D_{M}}{R_{M}}+w_{2} \log \frac{R_{M}}{B}=w_{1} \log \frac{D_{T}}{R_{T}}+w_{2} \log \frac{R_{T}}{B},
$$


solving this equation w.r.t. $\log D_{M}$ we have

$$
\begin{aligned}
\log D_{M} & =\log D_{T}+\left(1-\frac{w_{2}}{w_{1}}\right) \log R_{M} \\
& -\left(1-\frac{w_{2}}{w_{1}}\right) \log R_{T} .
\end{aligned}
$$

The WLLR model thus predicts that if we plot the Log of observer's matching disk settings versus the Log of the test ring luminance, the data should fall on a straight line having a slope $\beta=-\left(1-\frac{w_{2}}{w_{1}}\right) \in(-1,0)$, which is coherent with Rudd and Zemach's observations. The estimation of the ratio $\frac{w_{2}}{w_{1}}$ from their interpolated data for the four observers is: 0.361 (LT), 0.209 (IKZ), 0.277 (JL), and $0.343(\mathrm{AD})$.

If we set $c_{1} \equiv \log D_{T}+\left(1-\frac{w_{2}}{w_{1}}\right) \log R_{M}$ and $c_{2} \equiv$ $\frac{w_{2}}{w_{1}}-1$, then $\log D_{M}=c_{1}+c_{2} \log R_{T}=c_{1}+\log R_{T}^{c_{2}}$, so $D_{M}=10^{c_{1}+\log R_{T}^{c_{2}}}$, i.e.

$$
D_{M}=k R_{T}^{c_{2}}
$$

where $k=10^{c_{1}}$. This means that the brightness of $D_{M}$ is proportional to a power function of $R_{T}$ with exponent given by $\frac{w_{2}}{w_{1}}-1$, which, in the experiments of Rudd and Zemach, ranged from -0.639 to -0.791 .

A key assumption of edge integration models, as WLLR, is that the total achromatic color induction produced by a complex background is the sum of the individual induction effects produced by the luminance borders comprising that background. Rudd and Zemach performed experiments to directly test this assumption by predicting and then measuring the magnitude of the total induction effect produced by combining three circular edges located at different distances from the test disk.

This was done after first measuring the magnitudes of the induction effects produced by the individual edges. The combination of three inducing edges was produced by surrounding the test disk with two concentric rings as in Fig. 4 (courtesy of [2]).

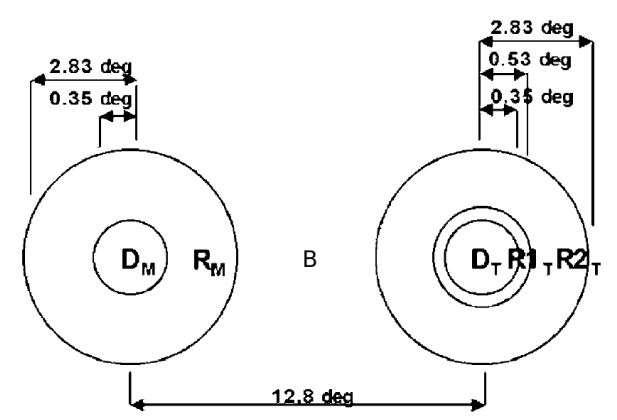

$R_{M}=3.96 \mathrm{~cd} / \mathrm{m}^{2} ; D_{M}=$ dependent variable; $D_{T}=1.02 \mathrm{~cd} / \mathrm{m}^{2}$
$R 1_{T}=6.31 \mathrm{~cd} / \mathrm{m}^{2} ; R 2_{T}=$ independent variable

Figure 4. Rudd-Wallach's experimental setting to test the additivity hypothesis underlying the WLLR model with two rings.

The observers in the two-ring experiment adjusted $D_{M}$ as a function of $R 2_{T}$ to achieve an achromatic color match between the test and matching disks. $R 1_{T}$ was fixed at the value $6.31 \mathrm{~cd} / \mathrm{m}^{2}\left(0.800 \mathrm{Log} \mathrm{cd} / \mathrm{m}^{2}\right)$ and $R 2_{T}$ was randomly varied from 2.54 to $6.31 \mathrm{~cd} / \mathrm{m}^{2}$ (0.405 to 0.800 $\left.\log c d / m^{2}\right)$ in six steps of equal units. $D_{M}$ and $R_{M}$ were fixed at the values $1.02 \mathrm{~cd} / \mathrm{m}^{2}\left(0.009 \mathrm{Log} \mathrm{cd} / \mathrm{m}^{2}\right)$ and $3.96 \mathrm{~cd} / \mathrm{m}^{2}\left(0.597 \mathrm{Log} \mathrm{cd} / \mathrm{m}^{2}\right)$ respectively. The width of the matching ring was $0.35 \mathrm{deg}$ (same as in the one-ring experiment).

According to the WLLR model, an achromatic color match should be achieved for this stimulus when this equation holds

$$
\begin{aligned}
w_{1} \log \frac{D_{M}}{R_{M}}+w_{3} \log \frac{R_{M}}{B} & =w_{1} \log \frac{D_{T}}{R 1_{T}}+w_{2} \log \frac{R 1_{T}}{R 2_{T}} \\
& +w_{3} \log \frac{R 2_{T}}{B},
\end{aligned}
$$

where $R 1_{T}$ is the luminance of the inner ring surrounding the test disk, $R 2_{T}$ is the luminance of the outer ring, and the subscripts on the induction weights indicate the distance between the border with which they are associated and the test disk perimeter, so $w_{1}$ stands for $w(0 \mathrm{deg}), w_{2}$ stands for $w(0.18 \mathrm{deg})$, and $w_{3}$ stands for $w(2.48 \mathrm{deg})$.

Solving this equation w.r.t. $\log D_{M}$ we have

$$
\begin{aligned}
\log D_{M} & =\log D_{T}+\left(1-\frac{w_{3}}{w_{1}}\right) \log R_{M} \\
& -\left(1-\frac{w_{2}}{w_{1}}\right) \log R 1_{T}+\left(\frac{w_{3}}{w_{1}}-\frac{w_{2}}{w_{1}}\right) \log R 2_{T} .
\end{aligned}
$$

This equation predicts that a Log-Log plot of the observer's matching disk settings versus outer ring luminance should form a straight line having the slope $\frac{w_{3}}{w_{1}}-\frac{w_{2}}{w_{1}}$. To test this prediction, they first measured the induction strength ratios $w_{3} / w_{1}$ and $w_{2} / w_{1}$ using eq. (7) in independent experiments in which the test disk was surrounded by a single ring only. Thus, accordingly to the WLLR model, the slopes of the lines depicting $\log D_{M}$ in function of $\log R 2_{T}$ should be given by the difference between the ratios $w_{3} / w_{1}$ and $w_{2} / w_{1}$ previously measured. The results are in accordance with the predictions.

\section{Generalization OF RUdD-ZEMACH'S MODEL TO ARBITRARY SPATIAL CONFIGURATIONS AND ITS VARIATIONAL INTERPRETATION}

It is natural to search for a generalization of RuddZemach's model valid for arbitrary spatial configurations and not only the very special one discussed in their experiments. To do that, let us introduce some convenient notation. We denote the visual spectrum as $\Lambda=(380,780) \mathrm{nm}$ and the 2-D projection of the spatial domain of the scene as $\Omega \subset \mathbb{Z}^{2} . x=\left(x_{1}, x_{2}\right)$ and $y=\left(y_{1}, y_{2}\right)$ will be the coordinates of two arbitrary points in $\Omega$.

The spectral luminance function $\ell: \Omega \times \Lambda \rightarrow(0,+\infty)$ can be decomposed in three luminance functions relative to the retinal cones, performing a weighted integration over $\Lambda$ with weights given by the spectral sensitivity 
functions $\bar{r}(\lambda), \bar{g}(\lambda), \bar{b}(\lambda)$ of the cones:

$$
\left\{\begin{array}{l}
L_{\bar{r}}(x)=\int_{\Lambda} \ell(x, \lambda) \bar{r}(\lambda) d \lambda, \\
L_{\bar{g}}(x)=\int_{\Lambda} \ell(x, \lambda) \bar{g}(\lambda) d \lambda, \\
L_{\bar{b}}(x)=\int_{\Lambda} \ell(x, \lambda) \bar{b}(\lambda) d \lambda .
\end{array}\right.
$$

Achromatic induction can be studied on the scalar components of $\vec{L}(x)=\left(L_{\bar{r}}(x), L_{\bar{g}}(x), L_{\bar{b}}(x)\right)$, that will be written as $L(x)$ without the subindex for a clearer notation.

With this notation in mind, the achromatic logarithmic brightness of an arbitrary point $x$ in Rudd-Zemach's model can be written as follows

$$
\Phi(x)=\int_{\Omega} w(x, y) \log \frac{L(y)}{L(x)} d y
$$

or, equivalently,

$$
\Phi(x)=\int_{\Omega} w(x, y)[\log L(y)-\log L(x)] d y .
$$

The following proposition provides a variational interpretation of this formula.

Proposition 1: Given the functional

$$
\begin{aligned}
E_{w}(\log L) & =\frac{1}{2} \int_{\Omega}\left(\log \frac{L_{0}(x)}{L(x)}\right)^{2} d x \\
& -\frac{1}{4} \iint_{\Omega^{2}} w(x, y)\left(\log \frac{L(y)}{L(x)}\right)^{2} d x d y
\end{aligned}
$$

where $L_{0}(x)$ is the photometric luminance of $x$ and must be interpreted as a constant, the Rudd-Zemach achromatic logarithmic brightness $\Phi(x)$ of an arbitrary point $x \in \Omega$ satisfied this equation

$$
\Phi(x)=\operatorname{argmin}_{\log L} E_{w}(\log L)+\log L_{0}(x) .
$$

The proof of this proposition is provided in the Appendix. Here, we would like to stress its interpretation: it is convenient to write the functional terms appearing in the energy as follows, $D(\log L) \equiv$ $\frac{1}{2} \int_{\Omega}\left(\log \frac{L_{0}(x)}{L(x)}\right)^{2} d x$ and call it quadratic dispersion term around the original logarithmic luminance, and $C_{w}(\log L) \equiv \frac{1}{4} \iint_{\Omega^{2}} w(x, y)\left(\log \frac{L(y)}{L(x)}\right)^{2} d x d y$ and call it local quadratic contrast term in the logarithmic domain. The minimization of $E_{w}(\log L)=D(\log L)-C_{w}(\log L)$ is reached through the simultaneous minimization of $D(\log L)$ and maximization of $C_{w}(\log L)$ (because of the minus sign in front of the contrast term). In particular, the constant term $\log L_{0}(x)$ is generated by the minimization of the dispersion term and it does not appear in RuddZemach's edge integration model, this is why it must be added in order to get $\Phi(x)$.

Thus, we can say that Rudd-Zemach's logarithmic brightness $\Phi(x)$ corresponds to the maximization of the local quadratic contrast in the logarithmic domain, where locality is given by the weight function $w(x, y)$.

\section{RELATIONSHiP BETWEEN RUDD-ZEMACH'S MODEL AND VARIATIONAL THEORIES OF PERCEPTUAL COLOR CORRECTION}

The result just obtained has many similarities with the theory of perceptually-inspired color correction developed in the papers [3], [7], [8]. In fact, also the functionals considered in these papers balance dispersion and local contrast to perform perceptual color correction, i.e. to modify the intensities $I(x)$ of pixels in digital images to approach the lightness and brightness of the points in the visual scene that pixels represent.

In [3], it has been proven that the most general perceptually-inspired energy functional $E_{w, \alpha, \beta}^{\varphi}(I)=$ $C_{w}^{\varphi}(I)+D_{\alpha, \beta}(I)$ that fulfills the basic HVS properties (local contrast enhancement, adjustment around the average value, color constancy and Weber-Fechner's law of contrast perception) is given by the sum these terms

$$
C_{w}^{\varphi}(I) \equiv \frac{1}{4} \iint_{\mathfrak{I}} w(x, y) \varphi\left(\frac{\min (I(x), I(y))}{\max (I(x), I(y))}\right) d x d y
$$

the functional parameter $\varphi$ being a strictly increasing positive function, and

$$
\begin{aligned}
D_{\alpha, \beta}(I) & \equiv \alpha \int_{\mathfrak{I}}\left(\mu \log \frac{\mu}{I(x)}-(\mu-I(x))\right) d x \\
& +\beta \int_{\mathfrak{I}}\left(I_{0}(x) \log \frac{I_{0}(x)}{I(x)}-\left(I_{0}(x)-I(x)\right)\right) d x .
\end{aligned}
$$

Let us discuss the meaning of $C_{w}^{\varphi}(I)$ and $D_{\alpha, \beta}(I)$, starting with the contrast term. Notice that the ratio defined by $\min (I(x), I(y)) / \max (I(x), I(y))$ is minimized when the minimum decreases and the maximum increases, which of course corresponds to a contrast stretching. Thus, minimizing an increasing function of that ratio will produce a contrast enhancement. Moreover, recalling that a global illuminant change can be represented as the transformation $I(x) \mapsto \lambda I(x), \lambda>0$, the contrast enhancement term will be unaffected by such a change, coherently with the color constancy property. Finally, it can be proven [3] that this definition of contrast is coherent with Weber-Fechner's law.

The dispersion term is based on the relative entropy distance [9] between $I$ and $\mu$ (first term) and between $I_{0}$ and $I$ (second term). Given the statistical interpretation of entropy, we can say that minimizing $D_{\alpha, \beta}(I)$ amounts to minimizing the disorder of intensity levels around the average $\mu$ and around the original data $I_{0}(x)$. Thus, $D_{\alpha, \beta}(I)$ serves as a global adaptation term.

By minimizing the energy $E_{w, \alpha, \beta}^{\varphi}$, e.g. through a gradient descent technique, we have the explicit algorithm implementation of this model:

$$
\partial_{t} \log I=-\delta E_{w, \alpha, \beta}^{\varphi}(I),
$$

$t$ being the evolution parameter and $\delta$ the first variation of $E$. For practical implementations this scheme must be discretized: choosing a finite evolution step $\Delta t>0$ and 
setting $I^{k}(x)=I_{k \Delta t}(x), k \in \mathbb{N}$, with $I^{0}(x)$ being the original image, we have

$$
I^{k+1}(x)=\frac{I^{k}(x)+\Delta t\left(\alpha \mu+\beta I^{0}(x)+\frac{1}{2} R_{w, I_{k}}^{\varphi}(x)\right)}{1+\Delta t(\alpha+\beta)} .
$$

As can be seen, $\alpha$ and $\beta$ represent the strength of the attachment to $1 / 2$ and to $I^{0}$, respectively, while $R_{w, I^{k}}^{\varphi}$ represents the contrast enhancement. When $\varphi \equiv \mathrm{id}$, the identity function, then

$$
\begin{aligned}
R_{I^{k}}^{\mathrm{id}}(x):= & \int_{\Omega} w(x, y) \frac{I^{k}(y)}{I^{k}(x)} H\left(I^{k}(x)-I^{k}(y)\right) d y \\
& -\int_{\Omega} w(x, y) \frac{I^{k}(x)}{I^{k}(y)} H\left(I^{k}(y)-I^{k}(x)\right) d y,
\end{aligned}
$$

$I^{k}$ is the image at the $k$-th step, $I^{0}$ is the original image, $H$ is the Heaviside step function, i.e. $H(t)=1$ when $t>0, H(t)=0$ when $t \leq 0$.

When $\alpha=\beta=0$, i.e. the adjustment coefficients are set to 0 , both equation (19) and (13) express the brightness computation as a generalized edge integration. The main difference is given by the fact that in (13) there appear logarithmic luminance values, while (19) is written in terms of pixel intensities. This difference is expected, since the transformation from real-world luminance to digital image intensity is a concave function, as it is the logarithm.

It would be interesting to perform psychophysical experiments to test if the attachment to the original luminance and to the average value $\mu$ play a significant role in improving the ability of Rudd-Zemach's model to measure achromatic induction.

\section{CONCLUSION}

We have discussed a variational interpretation of RuddZemach's model of achromatic induction, showing that it shares many similarities with recently built variational models for perceptually-inspired color correction.

We have proven that the edge integration formula on which Rudd-Zemach's is based can be interpreted as the maximization of local quadratic contrast of logarithmic luminances, where locality is given by induction weights quantitatively measured by Rudd and Zemach in [2].

This shows with even greater strength the exigence to extend Rudd-Zemach's experiments in many directions: to color stimuli, to a wider number of users, and to a larger luminance range, in order to have more reliable results that would allow building a proper metrology of chromatic and achromatic induction.

This would be highly beneficial, for example, for the quantitative determination of the weight function used in the color correction models [3], [7], [8]. Up to now, these functions are Gaussians whose standard deviation can be regulated by users to following their personal tastes and needs in the correction of images. However, in order to be as close as possible to human perception, a quantitative measure of induction weight, as that started by Rudd and Zemach, can set the parameter function $w(x, y)$ in the most adequate way in relation to achromatic and chromatic color perception.

\section{ACKNOWLEDGMENT}

The author acknowledges FUI project CEDCA.

\section{APPENDIX: PROOF OF PROPOSITION 1}

Let us compute the variation of the functional $E_{w}(\log L)=D(\log L)-C_{w}(\log L)$ with respect to $\log L$. To do this, it is convenient to write $\log L(x) \equiv$ $\tilde{L}(x)$. The variation of the first term of the functional is trivial to compute and gives:

$$
\delta D(\tilde{L}, J)=\int_{\Omega}\left(\tilde{L}_{0}(x)-\tilde{L}(x)\right) J(x) d x
$$

$J(x)$ being a generic functional perturbation of $\tilde{L}(x)$. The variation of the second term instead can be written as follows

$$
\begin{aligned}
\delta C_{w}(\tilde{L}, J) & =\frac{1}{2} \iint_{\Omega^{2}} w(x, y)[\tilde{L}(y)-\tilde{L}(x)] J(x) d x d y \\
& -\frac{1}{2} \iint_{\Omega^{2}} w(x, y)[\tilde{L}(y)-\tilde{L}(x)] J(y) d x d y
\end{aligned}
$$

Now, interchanging the role of the 'mute' variables $x$ and $y$ in the second integral and using the symmetry of the induction weight, we can write

$$
\delta C_{w}(\tilde{L}, J)=\iint_{\Omega^{2}} w(x, y)[\tilde{L}(y)-\tilde{L}(x)] J(x) d x d y .
$$

So, since $\delta E(\tilde{L}, J)=\delta D(\tilde{L}, J)-\delta C_{w}(\tilde{L}, J)$, we have that the Euler-Lagrange equation $\delta E(\tilde{L}, J)=0$ for all perturbations $J$, is satisfied if and only if the $\operatorname{argmin} \tilde{L}(x)$ satisfies

$$
\tilde{L}_{0}(x)-\tilde{L}(x)-\int_{\Omega} w(x, y)[\tilde{L}(y)-\tilde{L}(x)] d y=0,
$$

i.e.

$$
\operatorname{argmin}_{\log L}=\int_{\Omega} w(x, y)[\tilde{L}(y)-\tilde{L}(x)] d y-\tilde{L}_{0}(x) .
$$

Thus, the logarithmic brightness $\Phi(x)$ can be written as $\Phi(x)=\operatorname{argmin}_{\log L} E_{w}(\log L)+\log L_{0}(x)$ and so Proposition 1 is proven.

\section{REFERENCES}

[1] H. Wallach, "Brightness constancy and the nature of achromatic colors," Journal of Experimental Psychology, vol. 38, no. 3, pp. 310-324, 1948.

[2] M. Rudd and I. Zemach, "Quantitive properties of achromatic color induction: An edge integration analysis," Vision Research, vol. 44, pp. 971-981, 2004.

[3] R. Palma-Amestoy, E. Provenzi, M. Bertalmío, and V. Caselles, "A perceptually inspired variational framework for color enhancement," IEEE Transactions on Pattern Analysis and Machine Intelligence, vol. 31, no. 3, pp. 458-474, 2009. 
[4] E. Land and J. McCann, "Lightness and Retinex theory," Journal of the Optical Society of America, vol. 61, no. 1, pp. 1-11, Jan. 1971.

[5] E. Provenzi, L. De Carli, A. Rizzi, and D. Marini, "Mathematical definition and analysis of the retinex algorithm," Journal of the Optical Society of America A, vol. 22, no. 12, pp. 2613-2621, Dec. 2005.

[6] H. Wallach, "The perception of neutral colors," Scientific American, vol. 208, pp. 107-116, 1963.

[7] M. Bertalmío, V. Caselles, E. Provenzi, and A. Rizzi, "Perceptual color correction through variational techniques," IEEE Trans. on Image Processing, vol. 16, pp. 1058-1072, 2007.

[8] M. Bertalmío, V. Caselles, and E. Provenzi, "Issues about the retinex theory and contrast enhancement," International Journal of Computer Vision, vol. 83, pp. 101-119, 2009.

[9] L. Ambrosio, N. Gigli, and G. Savaré, Gradient flows in metric spaces and in the space of probability measures. Lectures in Mathematics, Birkhauser, 2005. 